\title{
VOLUMES OF TUBES ABOUT COMPLEX SUBMANIFOLDS OF COMPLEX PROJECTIVE SPACE
}

\begin{abstract}
ALFRED GRAY
ABStract. Simple formulas in terms of Chern classes are given for the volume of a tube about a Kähler submanifold of a space of constant holomorphic sectional curvature. A comparison theorem which generalizes these formulas is also given. Tubes about complete intersections in complex projective space are studied in detail.
\end{abstract}

1. Introduction. Let $M$ be a complete Kähler manifold whose sectional curvature is bounded from below or from above, and let $P$ be a topologically embedded Kähler submanifold of $M$ with compact closure. I shall give inequalities for the volume $V_{p}^{M}(r)$ of a tube of radius $r$ about $P$ in terms of the bounds on the sectional curvature of $M$ (Theorem 6.1). These results generalize the Bishop-Günther comparison theorem for the volumes of geodesic balls [BC, p. 256] as well as the Weyl tube formula [W].

The case when $M$ has nonnegative or nonpositive sectional curvature has been treated in [GR3, 4, 5], so this article is mainly devoted to the case when $M$ has sectional curvature bounded above or below by a nonzero constant. Perhaps the most interesting special case is that of a compact complex submanifold $P$ of complex projective space $\mathbf{C} P^{n}(\lambda)$. (Here the Fubini-Study metric is used for $\mathbf{C} P^{n}(\lambda)$; it is chosen so that the holomorphic sectional curvature of $\mathbf{C} P^{n}(\lambda)$ is $4 \lambda$ ). Let $q$ be the complex dimension of $P$ and let $\gamma=1+\gamma_{1}+\cdots+\gamma_{q}$ be the total Chern form of $P$. Write $\gamma(t)=1+t \gamma_{1}+\cdots+t^{q} \gamma_{q}$ and let $\gamma(t)=\prod_{a=1}^{q}\left(1+t x_{a}\right)$ be its formal factorization. Denote by $F$ the Kähler form of $\mathbf{C} P^{n}(\lambda)$ (or of $P$ ).

THEOREM 1.1. Suppose $r>0$ is not larger than the distance between $P \subset \mathrm{CP}^{n}(\lambda)$ and its nearest focal point. Then

$$
V_{P}^{\mathbf{C} P^{n}(\lambda)}(r)=\frac{1}{n !} \int_{P} \prod_{a=1}^{q}\left(1-\frac{\lambda}{\pi} F+x_{a}\right) \wedge\left(\frac{\pi}{\lambda} \sin ^{2} \sqrt{\lambda} r+\cos ^{2} \sqrt{\lambda} r F\right)^{n} .
$$

Note that on the right-hand side of (1.1) all terms not of degree $2 q$ must be discarded before the integration is carried out. Formula (1.1) can be written somewhat more explicitly as

$$
V_{P}^{\mathbf{C} P^{n}(\lambda)}(r)=\frac{1}{n !} \int_{P} \sum_{a=1}^{q}\left(1-\frac{\lambda}{\pi} F\right)^{q-a} \wedge \gamma_{a} \wedge\left(\frac{\pi}{\lambda} \sin ^{2} \sqrt{\lambda} r+\cos ^{2} \sqrt{\lambda} r F\right)^{n}
$$

Received by the editors December 5, 1983.

1980 Mathematics Subject Classification. Primary 53C55. 
Theorem (1.1) can be used to calculate the volumes of tubes about many interesting complex submanifolds of $\mathbf{C} P^{n}(\lambda)$, for example complete intersections (see also Remark (5) below).

COROllaRY 1.2. Let $P=P^{a_{1} \cdots a_{n-q}}(\lambda) \subset \mathbf{C} P^{n}(\lambda)$ be a complete intersection, defined as the simultaneous zeros of polynomials of degrees $a_{1} \cdots a_{n-q}$. Assume that $r>0$ is not larger than the distance from $P$ to its nearest focal point. Then

$$
V_{P}^{\mathbf{C} P^{n}(\lambda)}(r)=\frac{1}{n !} \int_{P} \frac{\left((\pi / \lambda) \sin ^{2} \sqrt{\lambda} r+\cos ^{2} \sqrt{\lambda} r F\right)^{n}}{(1(\lambda / \pi) F) \prod_{a=1}^{n-q}\left(1+\left(\left(a_{c}-1\right) \lambda / \pi\right) F\right)} .
$$

There is a formula for the volume of $p^{a_{1} \cdots a_{n-q}}(\lambda)[\mathbf{M U}$, p. 89, K]:

$$
\operatorname{vol}\left(P^{a_{1} \cdots a_{n-q}}(\lambda)\right)=\frac{a_{1} \cdots a_{n-q} \pi^{q}}{q ! \lambda^{q}} .
$$

When (1.4) is combined with (1.3) a more explicit formula for $V_{P}^{C P^{n}(\lambda)}(r)$ results.

Corollary 1.3. Assume the hypotheses of Corollary 1.2. Then

$$
V_{P}^{\mathbf{C} P^{n}(\lambda)}(r)=\frac{1}{n !}\left(\frac{\pi}{\lambda}\right)^{n} \sum_{c=0}^{q}\left(\begin{array}{l}
n \\
c
\end{array}\right)\left(\sin ^{2} \sqrt{\lambda} r\right)^{n-c}\left(\cos ^{2} \sqrt{\lambda} r\right)^{c} \prod_{i=1}^{n-q}\left\{1-\left(1-a_{i}\right)^{q-c+1}\right\} .
$$

In particular, the formula for the volume of a tube of radius $r$ about a complex hypersurface of degree $d$ is

$$
V_{P}^{\mathrm{C} P^{n}(\lambda)}(r)=\frac{1}{n !}\left(\frac{\pi}{\lambda}\right)^{n}\left\{1-\left(1-d \sin ^{2} \sqrt{\lambda} r\right)^{n}\right\} .
$$

(Also of interest of course is the special case $q=0$; this is the formula (already known to Study [S]) for the volume of a geodesic ball in $\mathbf{C} P^{n}(\lambda), V_{m}^{\mathbf{C} P^{n}(\lambda)}(r)=$ $(1 / n !)\left((\pi / \lambda) \sin ^{2} \sqrt{\lambda} r\right)^{n}$.)

COROLlARY 1.4. The volume of a tube of radius $r$ about a complete intersection in $\mathrm{CP}^{n}(\lambda)$ depends only on $\lambda, r$ and the degrees of the polynomials defining the complete intersection.

REMARKS. (1) There are formulas corresponding to Theorem 1.1 for tubes about complex submanifolds of $\mathbf{C}^{n}$ or $\mathbf{C} H^{n}(\lambda)$ (where $\mathbf{C} H^{n}(\lambda)$ denotes complex hyperbolic space with holomorphic sectional curvature $-4 \lambda, \lambda>0)$. However, in these cases complex submanifolds of positive dimension are necessarily noncompact. Nevertheless tube formulas are meaningful when the closure $\bar{P}$ is compact. The corresponding formulas are

$$
\begin{gathered}
V_{P}^{\mathbf{C}^{n}}(r)=\frac{1}{n !} \int_{P} \gamma \wedge\left(\pi r^{2}+F\right)^{n} \\
V_{P}^{\mathbf{C} H^{n}(\lambda)}(r)=\frac{1}{n !} \int_{P} \prod_{a=1}^{q}\left(1-\frac{\lambda}{\pi} F+x_{a}\right) \wedge\left(\frac{\pi}{\lambda} \sinh ^{2} \sqrt{\lambda} r+\cosh ^{2} \sqrt{\lambda} r F\right)^{n} .
\end{gathered}
$$

(For (1.6) see also [GR5]; some information about $V_{P}^{\mathbf{C}^{n}}(r)$ is also given [GS].)

(2) Let $P$ be a compact Kähler submanifold of a compact Kähler manifold $M$. Then $P$ defines an element $[P]$ in the homology ring $H_{*}(M, \mathbf{R})$. Hence (see for 
example [MS, p. 196] and [GH, p. 54]), one can use cap products and de Rham's theorem to show that there is a closed differential form $\phi$ in $M$ such that the class $[\phi] \in H^{*}(M, \mathbf{R})$ is dual to $P$. This means that for any closed form $\alpha$ on $M$ one has

$$
\alpha[P]=\int_{P} \alpha=\int_{M} \alpha \wedge \phi=(\alpha \wedge \phi)[M] .
$$

From (1.8) it follows that (1.1) can be rewritten as

$$
V_{P}^{\mathbf{C} P^{n}(\lambda)}(r)=\frac{1}{n !} \int_{\mathbf{C} P^{n}(\lambda)} \phi \wedge \prod_{a=1}^{q}\left(1-\frac{\pi}{\lambda} F+x_{a}\right) \wedge\left(\frac{\pi}{\lambda} \sin ^{2} \sqrt{\lambda} r+\cos ^{2} \sqrt{\lambda} r F\right)^{n} .
$$

In the case that $\operatorname{dim}_{\mathbf{C}} M=\operatorname{dim}_{\mathbf{C}} P+1$ one has in fact that

$$
[\phi]=\left[\gamma_{1}(\nu(P))\right]=\text { the first Chern class of the normal bundle of } P \text { in } M \text {. }
$$

(See for example [MS, p. 196].) Thus for a complex intersection $P=P^{a_{1} \cdots a_{n-q}}(\lambda)$ in $\mathbf{C} P^{n}(\lambda)$ the dual class $[\phi]$ can be computed by induction. It turns out that

$$
[\phi]=a_{1} \cdots a_{n-q}\left[((\lambda / \pi) F)^{n-q}\right] .
$$

Thus using (1.8) and (1.9) it follows that (1.3) can be reformulated as

$$
(1.3)^{\prime} \quad V_{P}^{\mathbf{C} P^{n}(\lambda)}(r)=\frac{1}{n !} \int_{\mathbf{C} P^{n}(\lambda)} \frac{\left((\pi / \lambda) \sin ^{2} \sqrt{\lambda} r+\cos ^{2} \sqrt{\lambda} r F\right)^{n}((\lambda / \pi) F)^{n-q}}{(1-(\lambda / \pi) F) \prod_{a=1}^{q}\left\{1+\left(\left(a_{c}-1\right) \lambda / \pi\right) F\right\}} .
$$

(3) Note that for any Kähler manifold $P$ and any integer $n$ it is possible to define

$$
V_{P}^{\mathbf{C} P^{n}(\lambda)}(r), \quad V_{P}^{\mathbf{C}^{n}}(r) \quad \text { and } V_{P}^{\mathbf{C} H^{n}(\lambda)}(r)
$$

by formulas (1.1), (1.6) and (1.7). All that is required is that the relevant integrals converge. Thus the expressions in (1.10) make sense formally even when $P$ is not embedded in $\mathbf{C} P^{n}(\lambda), \mathbf{C}^{n}$ or $\mathbf{C} H^{n}(\lambda)$. For example when $n=0$ and $P$ is compact all of the expressions in (1.10) coincide with the Euler characteristic of $P$.

(4) To what extent do the volume functions in (1.10) depend on the ambient space $\mathbf{C} P^{n}(\lambda), \mathbf{C}^{n}$ or $\mathbf{C H}^{n}(\lambda)$ ? Recall (see for example [BGM, p. 106]) that a Kähler deformation consists of a change of Kähler form from $F$ to $F+i d^{\prime} d^{\prime \prime} f$, where $f$ is any real-valued (differentiable) function.

THEOREM 1.5. Let $P$ be a compact Kähler manifold with Kähler form $F$. Define $V_{P}^{\mathbf{C} P^{n}(\lambda)}(r), V_{P}^{\mathbf{C}^{n}}(r)$ and $V_{P}^{\mathbf{C} H^{n}(\lambda)}(r)$ by formulas (1.1), (1.6) and (1.7). Then these functions are the same for all Kähler deformations. In particular, if $(Q, M)$ is a Kähler deformation of $\left(P, \mathbf{C} P^{n}(\lambda)\right)$, then

$$
V_{Q}^{M}(r)=V_{P}^{C P^{n}(\lambda)}(r)
$$

The proof of Theorem 1.5 is elementary (given formulas (1.1), (1.6) and (1.7)) and is given in [GR5]. 
(5) It is interesting to write out formulas (1.1) and (1.6) for certain simple Kähler manifolds $P$. If $P$ is a compact Riemann surface with Euler characteristic $\chi(P)$, then

$$
\begin{aligned}
& V_{P}^{\mathbf{C} P^{n}(\lambda)}(r)= \frac{1}{(n-1) !}\left(\frac{\pi}{\lambda} \sin ^{2} \sqrt{\lambda} r\right)^{n-1} \\
& \cdot\left\{\left(1-\frac{n+1}{n} \sin ^{2} \sqrt{\lambda} r\right) \operatorname{vol} P+\frac{\pi \sin ^{2} \sqrt{\lambda} r}{n \lambda} \chi(P)\right\}, \\
& V_{P}^{\mathbf{C}^{n}}(r)=\frac{\left(\pi r^{2}\right)^{n-1}}{(n-1) !}\left\{\operatorname{vol} P+\frac{\pi r^{2}}{n} \chi(P)\right\} .
\end{aligned}
$$

These formulas are simple consequences of the fact that $\int_{P} \gamma_{1}=\chi(P)$.

Similarly, for a compact complex surface the tube formulas are

$$
\begin{aligned}
V_{P}^{\mathbf{C} P^{n}(\lambda)}(r)= & \frac{1}{(n-2) !}\left(\frac{\pi}{\lambda} \sin ^{2} \sqrt{\lambda} r\right)^{n-2} \\
& \cdot\left\{\left(1-\frac{2(n+1)}{n-1} \sin ^{2} \sqrt{\lambda} r+\frac{(n+1)(n+2)}{n(n-1)} \sin ^{4} \sqrt{\lambda} r\right) \operatorname{vol} P\right. \\
& \quad+\frac{1}{n-1}\left(\frac{\pi}{\lambda} \sin ^{2} \sqrt{\lambda} r\right)\left(1-\frac{n+1}{n} \sin ^{2} \sqrt{\lambda} r\right)\left(F \wedge \gamma_{1}\right)[P] \\
& \left.\quad+\frac{1}{n(n+1)}\left(\frac{\pi}{\lambda} \sin ^{2} \sqrt{\lambda} r\right)^{2} \chi(P)\right\} \\
V_{P}^{\mathbf{C}^{n}}(r)= & \frac{\left(\pi r^{2}\right)^{n-2}}{(n-2) !}\left\{\operatorname{vol} P+\frac{\pi r^{2}}{n-1}\left(F \wedge \gamma_{1}\right)[P]+\frac{\left(\pi r^{2}\right)^{2}}{n(n-1)} \chi(P)\right\} .
\end{aligned}
$$

Another case of interest is that of a flat submanifold $P^{q} \subset \mathbf{C} P^{n}(\lambda)$. Then

$$
V_{P}^{\mathbf{C} P^{n}(\lambda)}(r)=\frac{q !}{n !}\left(\frac{\pi}{\lambda} \sin ^{2} \sqrt{\lambda} r\right)^{n-q} \sum_{c=0}^{q}\left(\begin{array}{c}
n \\
q-c
\end{array}\right)\left(\begin{array}{c}
n+c \\
c
\end{array}\right)(-1)^{c}\left(\sin ^{2} \sqrt{\lambda} r\right)^{c} \text { vol } P,
$$

Similarly, when $P^{q} \subset \mathbf{C} P^{n}(\lambda)$ is totally geodesic,

$$
V_{P}^{C P^{n}(\lambda)}(r)=\frac{1}{(n-q) !}\left(\frac{\pi}{\lambda} \sin ^{2} \sqrt{\lambda} r\right)^{n-q} \operatorname{vol}(P) .
$$

2. Four equations describing the geometry of tubes. Let $M$ be a complete Riemannian manifold of dimension $n$ and let $P \subset M$ be a topologically embedded submanifold which is relatively compact. In this section results of [GR3] are summarized; in subsequent sections they will be specialized to Kähler manifolds.

Let $t \rightarrow \gamma(t)$ be a unit speed geodesic in $M$ normal to $P$ with $\gamma(0)=p \in P$. Assume that $t \geqslant 0$ is less than the distance between $P$ and its nearest focal point. Denote by $S(t)$ the second fundamental form at the point $\gamma(t)$ of the tubular hypersurface at a distance $t$ from $P$. Also let $R(t): M_{\gamma(t)} \rightarrow M_{\gamma(t)}$ be the linear transformation defined by $\langle R(t), x, y\rangle=R_{\gamma^{\prime}(t) x \gamma^{\prime}(t) y}^{M}$, where $\langle$,$\rangle and R^{M}$ are the metric and curvature tensor fields of $M, M_{\gamma(t)}$ denotes the tangent space to $M$ at $\gamma(t)$, and $x, y \in M_{\gamma(t)}$.

Proposition 2.1. The following Riccati equation holds:

$$
S^{\prime}(t)=S(t)^{2}+R(t) \text {. }
$$


The study of (2.1) is essentially equivalent to the study of Jacobi fields near $P$. Equation (2.1) has the advantage that it yields direct information about the principal curvatures of the tubular hypersurfaces.

Next let $\omega$ be a Riemannian volume form defined near $P$ with $\|\omega\|=1$, and let $\left(x_{1}, \ldots, x_{n}\right)$ be a system of Fermi coordinates (cf. [GR3]) such that

$$
\omega\left(\frac{\partial}{\partial x_{1}} \wedge \cdots \wedge \frac{\partial}{\partial x_{n}}\right)(\gamma(t))>0 .
$$

For $u \in P^{\perp}$ with $\|u\|=1$, put

$$
\theta_{u}(t)=\omega\left(\frac{\partial}{\partial x_{1}} \wedge \cdots \wedge \frac{\partial}{\partial x_{n}}\right)(\gamma(t)) .
$$

Then $\theta_{u}(t)$ measures the ratio of the infinitesimal volume element of the normal bundle of $P$ to the infinitesimal volume element of $M$.

Proposition 2.2. $\theta_{u}(0)=1$ and

$$
\theta_{u}^{\prime}(t) / \theta_{u}(t)=-((n-q-1) / t+\operatorname{tr} S(t)) .
$$

Let $V_{P}^{M}(r)$ be the volume of a tube of radius $r$ about $P$; thus

$$
\begin{aligned}
& V_{P}^{M}(r)=\operatorname{vol}\{m \in M \mid \text { there exists a geodesic } \gamma \text { with length } \\
& \qquad L(\gamma) \leqslant r \text { from } m \text { to } P \text { meeting } P \text { orthogonally }\} .
\end{aligned}
$$

(See [GR3] for details.) Also let $S^{n-q-1}(1)$ be the unit sphere in $P_{p}^{\perp}$.

Proposition 2.3. If $0 \leqslant r \leqslant$ distance from $P$ to its nearest focal point, then

$$
V_{P}^{M}(r)=\int_{0}^{r} \int_{P} \int_{S^{n-q-1}(1)} t^{n-q-1} \theta_{u}(t) d u d P d t
$$

By means of (2.1), (2.2) and (2.3) the tube volume $V_{P}^{M}(r)$ is expressed in terms of the second fundamental forms of the tubular hypersurfaces.

There is a fourth equation of a different sort that is relevant to the estimation of $V_{P}^{M}(r)$ in terms of curvature, and also to the Weyl tube formula. Let $T_{u}: P_{p} \rightarrow P_{p}$ denote the second fundamental form of the submanifold $P$ at $p \in P$ in the direction $u \in P_{p}^{\perp}$, and consider the function

$$
s \rightarrow \operatorname{det}\left(I-s T_{u}\right)
$$

In general this function will depend on the immersion of $P$ in $M$. It is remarkable, however, that the average of $\operatorname{det}\left(I-s T_{u}\right)$ over $S^{n-q-1}(1) \subset P_{p}^{\perp}$ is expressible in terms of the curvature of $P$ and $M$; hence this average is independent of the immersion, for example when $M$ is a homogeneous space. More precisely,

PROPOSITION 2.4.

$$
\begin{aligned}
& \int_{S^{n-q-1}(1)} \operatorname{det}\left(I-s T_{u}\right) d u \\
&=\frac{2 \pi^{(n-q) / 2}}{\Gamma((n-q) / 2)} \sum_{c=0}^{[q / 2]} \frac{C^{2 c}\left(R^{p}-R^{M}\right)_{p}^{c} s^{2 c}}{c !(2 c) !(n-q) \cdots(n-q+2 c-2)}
\end{aligned}
$$


Here $R^{P}$ denotes the curvature operator of $P$ and $R^{M}$ denotes the restriction to $P$ of the curvature operator of $M$. Then $\left(R^{P}-R^{M}\right)^{c}$ is the $c$ th power of $R^{P}-R^{M}$ and $C^{2 c}\left(R^{P}-R^{M}\right)^{c}$ is its complete contraction. (See for example [GR] and the next section.)

Proposition 2.4 is a key step necessary to establish Weyl's tube formula [W]. For the case $M=\mathbf{R}^{n}$ the function $\theta_{u}(t)$ and $\operatorname{det}\left(I-t T_{u}\right)$ coincide. Consequently Propositions 2.1-2.4 can be used to establish Weyl's tube formula for the volume of a tube about a submanifold of $\mathbf{R}^{n}$. Similarly, for a space of constant curvature $\lambda>0$, one has

$$
\theta_{u}(t)=\left(\frac{\sin \sqrt{\lambda} r}{\sqrt{\lambda} t}\right)^{n-q-1} \operatorname{det}\left(\cos \sqrt{\lambda} t-\frac{\sin \sqrt{\lambda} t}{\sqrt{\lambda}} T_{u}\right)
$$

and again a tube formula obtains. More generally, when the sectional curvature of $M$ satisfies $K^{M} \geqslant \lambda$ or $K^{M} \leqslant \lambda,(2.5)$ still holds with " = " replaced by " $\leqslant$ " or " $\geqslant$ ". From this estimates for $V_{P}^{M}(r)$ follow. (See [GR3] for details.)

For submanifolds of $\mathbf{C} P^{n}(\lambda)$ it is also possible to derive tube formulas. This is the subject of the following sections.

3. Chern forms and curvature. Let $P$ be any almost Hermitian manifold of complex dimension $q$ and let $R$ be any tensor field on $P$ that has all of the symmetries of the curvature tensor field of a Kähler manifold. (Later $R$ will be taken to be $R^{P}-$ $R^{\mathrm{C} P^{n}(\lambda)}$.) Let $\left\{E_{1} J E_{1} \cdots E_{q} J E_{q}\right\}$ be a local holomorphic orthonormal frame field on $P$. Then the complex curvature forms of $R$ with respect to this frame field are the 2-forms $\Xi_{a b}$ defined by

$$
\Xi_{a b}(X \wedge Y)=R_{E_{a} E_{b} X Y}-\sqrt{-1} R_{E_{a} J E_{b} X Y}
$$

for complex vector fields $X, Y$ on $P$. By definition the total Chern form of $R$ is

$$
\gamma(R)=1+\gamma_{1}(R)+\cdots+\gamma_{q}(R)=\operatorname{det}\left(\delta_{a b}+(\sqrt{-1} / 2 \pi) \Xi_{a b}\right)
$$

where $\gamma_{c}(R)$ is a $2 c$-form on $P$.

It is also possible to define the $c$ th power of $R$ (see for example [GR1]). The definition can be given inductively via the formulas $R^{0}=1$ and

$$
\begin{aligned}
& R^{c}\left(X_{1} \wedge \cdots \wedge X_{2 c}\right)\left(Y_{1} \wedge \cdots \wedge Y_{2 c}\right) \\
& \quad=\sum_{\substack{i j k l=1 \\
i<j, k<l}}^{2 c}(-1)^{i+j+k+l} R_{X_{i} X_{j} Y_{k} Y_{l}} R^{l-1}\left(X_{1} \wedge \cdots \wedge \hat{X}_{i} \wedge \cdots \wedge \hat{X}_{j} \wedge \cdots \wedge X_{2 c}\right) \\
& \quad \cdot\left(Y_{1} \wedge \cdots \wedge \hat{Y}_{k} \wedge \cdots \wedge \hat{Y}_{l} \wedge \cdots \wedge Y_{2 c}\right) .
\end{aligned}
$$

Then the complete contraction of $R^{c}$ is

$$
C^{2 c} R^{c}=\sum_{a_{1} \cdots a_{c}=1}^{2 q} R^{c}\left(E_{a_{1}} \wedge \cdots \wedge E_{a_{2 c}}\right)\left(E_{a_{1}} \wedge \cdots \wedge E_{a_{2 c}}\right)
$$

where $\left\{E_{1} \cdots E_{2 q}\right\}$ is any local orthonormal frame field on $P$.

According to [GR4] there is a relation between the Chern forms and the $c$ th powers of $R$.

Proposition 3.1. For $0 \leqslant c \leqslant q$,

$$
q !(q-c) ! C^{2 c} R^{c}=c !(2 c) !(2 \pi)^{c}\left\langle\gamma_{c}(R) \wedge F^{q-c}, F^{q}\right\rangle .
$$


Next write

$$
\gamma(R)(t)=1+t \gamma_{1}(R)+\cdots+t^{q} \gamma_{q}(R)
$$

and let

$$
\gamma(R)(t)=\prod_{a=1}^{q}\left(1+t x_{a}\right)
$$

be its formal factorization. Also put

$$
\hat{\gamma}(R)(t)=\prod_{a=1}^{q}\left(1-\frac{\lambda}{\pi} F+t x_{a}\right), \quad \hat{\gamma}(R)=\hat{\gamma}(R)(1) .
$$

Then it is easy to see that formally

Proposition 3.2.

$$
\hat{\gamma}(R)(t)=\left(1-\frac{\lambda}{\pi} F\right)^{q} \gamma(R)\left(\frac{t}{1-(\lambda / \pi) F}\right) .
$$

The main case of interest is when $P$ is a complex submanifold of a space of constant holomorphic sectional curvature, say $\mathbf{C} P^{n}(\lambda)$, and $R=R^{P}-R^{\mathbf{C P}^{n}(\lambda)}$. According to [GR4] there is a relation between $\gamma\left(R^{P}-R^{\mathbf{C} P^{n}(\lambda)}\right)$ and $\gamma\left(R^{P}\right)$.

Proposition 3.3.

$$
\gamma\left(R^{P}-R^{\mathbf{C} P^{n}(\lambda)}\right)=\sum_{a=0}^{q}\left(1+\frac{\lambda}{\pi} F\right)^{q-a+1} \wedge \gamma_{a}\left(R^{P}\right) .
$$

Therefore,

Proposition 3.4. If $P$ is a complex submanifold of $\mathbf{C} P^{n}(\lambda)$, then

$$
\frac{\gamma\left(R^{P}-R^{\mathrm{CP}(\lambda)}\right)}{1-(\lambda / \pi) F}=\prod_{a=1}^{q}\left(1-\frac{\lambda}{\pi} F+x_{a}\right) .
$$

Proof. From Proposition 3.3 it follows that

$$
\begin{aligned}
\frac{\gamma\left(R^{P}-R^{C P^{n}(\lambda)}\right)}{1-(\lambda / \pi) F} & =\sum_{a=0}^{q}\left(1-\frac{\lambda}{\pi} F\right)^{q-a} \wedge \gamma_{a}\left(R^{P}\right) \\
& =\left(1-\frac{\lambda}{\pi} F\right)^{q}+\left(1-\frac{\lambda}{\pi} F\right)\left(x_{1}+\cdots+x_{q}\right)+\cdots+x_{1} \cdots x_{q} \\
& =\prod_{a=1}^{q}\left(1-\frac{\lambda}{\pi} F+x_{a}\right) .
\end{aligned}
$$

4. Tubes about complex submanifolds of complex projective space. Although (2.1) is too complicated to solve for general Riemannian manifolds, it can be solved for complex submanifolds of $\mathbf{C} P^{n}(\lambda)$. To this end, let $\left\{e_{1} e_{1} \cdots e_{n} e_{n}^{*}\right\}$ be an orthonormal basis of the tangent space $M_{P}$ such that $J e_{1}=e_{1}^{*}, e_{q+1}=\gamma^{\prime}(0)$ and $e_{1} e_{1}^{*} \cdots e_{q} e_{q}^{*}$ are tangent to $P$. It may be assumed that $e_{1} e_{q}^{*} \cdots e_{q} e_{q}^{*}$ diagonalize the second fundamental form $T_{u}$ of $P$ where $u=e_{q+1}=\gamma^{\prime}(0)$. Let $\kappa_{1}(0), \kappa_{1}^{*}(0) \cdots \kappa_{q}(0), \kappa_{q}^{*}(0)$ be the corresponding eigenvalues. Extend $e_{1} e_{1}^{*} \cdots e_{n} e_{n}^{*}$ to orthonormal vector fields $E_{1} E_{1}^{*} \cdots E_{n} E_{n}^{*}$ along $\gamma$ so that at each point $E_{q+1}(t)=\gamma^{\prime}(t)$ and the other $E_{\alpha}(t)$ 
diagonalize $S(t)$. Now the curvature tensor of $\mathbf{C} P^{n}(\lambda)$ is given by the formula

$$
\begin{aligned}
R_{w x y z}=\lambda\{\langle w y\rangle\langle x z\rangle-\langle w z\rangle\langle x y\rangle & \\
& +\langle J w y\rangle\langle J x z\rangle-\langle J w z\rangle\langle J x y\rangle+2\langle J w x\rangle\langle J y z\rangle\},
\end{aligned}
$$

where wxyz are tangent vectors to $\mathbf{C} P^{n}(\lambda)$. From (2.1) and (4.1) it follows that

$$
\left\{\begin{array}{l}
\kappa_{\alpha}^{\prime}=\kappa_{\alpha}^{2}+\lambda, \quad \alpha \neq q+1,(q+1)^{*}, \\
\kappa_{(q+1)^{*}}^{\prime}=\kappa_{(q+1)^{*}}^{2}+4 \lambda .
\end{array}\right.
$$

Here $\left|\kappa_{a}(0)\right|<\infty$ for $a=1 \cdots q^{*}$ while $\kappa_{i}(0)=-\infty$ for $i=(q+1)^{*} \cdots n^{*}$. Thus (4.2) can be solved explicitly:

$$
\left\{\begin{array}{l}
\kappa_{a}(t)=-\frac{d}{d t} \ln \left(\cos \sqrt{\lambda} t-\frac{\kappa_{a}(0)}{\sqrt{\lambda}} \sin \sqrt{\lambda} t\right) \text { for } a=1 \cdots q^{*}, \\
\kappa_{i}(t)=-\frac{d}{d t} \ln (\sin \sqrt{\lambda} t) \text { for } i=q+2 \cdots n^{*}, \\
\kappa_{(q+1)^{*}}(t)=-\frac{d}{d t} \ln (\sin 2 \sqrt{\lambda} t) .
\end{array}\right.
$$

From (4.3) it follows that

$$
\begin{aligned}
\operatorname{tr} S(t)=-\frac{d}{d t}\left\{\sum_{a=1}^{q^{*}} \ln \left(\cos \sqrt{\lambda} t-\frac{\kappa_{a}(0)}{\sqrt{\lambda}} \sin \sqrt{\lambda} t\right)\right. \\
\quad+2(n-q-1) \ln \sin \sqrt{\lambda} t+\ln \sin 2 \sqrt{\lambda} t\} .
\end{aligned}
$$

Because of the explicit form of (4.4), equation (2.2) can be solved explicitly. The result is

$$
\theta_{u}(t)=\left(\frac{\sin \sqrt{\lambda} t}{\sqrt{\lambda} t}\right)^{2 n-2 q-1} \cos \sqrt{\lambda} t \prod_{a=1}^{q^{*}}\left(\cos \sqrt{\lambda} t-\frac{\kappa_{a}(0)}{\sqrt{\lambda}} \sin \sqrt{\lambda} t\right) .
$$

Now $\kappa_{a}(0)+\kappa_{a^{*}}(0)=0$ for $a=1 \cdots q$, because $P$ is a complex submanifold of $C P^{n}(\lambda)$. Thus (4.5) reduces to

$$
\begin{aligned}
\theta_{u}(t) & =\left(\frac{\sin \sqrt{\lambda} t}{\sqrt{\lambda} t}\right)^{2 n-2 q-1} \cos \sqrt{\lambda} t \prod_{a=1}^{q}\left(\cos ^{2} \sqrt{\lambda} t-\frac{\kappa_{a}(0)}{\sqrt{\lambda}} \sin ^{2} \sqrt{\lambda} t\right) \\
& =\left(\frac{\sin \sqrt{\lambda} t}{\sqrt{\lambda} t}\right)^{2 n-2 q-1}(\cos \sqrt{\lambda} t)^{2 q+1} \operatorname{det}\left(I-\frac{\tan \sqrt{\lambda} t}{\sqrt{\lambda}} T_{u}\right) .
\end{aligned}
$$

It is now possible to derive a formula for $V_{P}^{\mathbf{C} P(\lambda)}(r)$ using equations (2.3), (2.4) and (4.6).

Lemma 4.1. For $0 \leqslant t \leqslant$ the distance between $P$ and its nearest focal point,

$$
\begin{aligned}
\frac{d}{d t} V_{P}^{C P^{n}(\lambda)}(t)= & 2 \pi^{n-q}(\cos \sqrt{\lambda} t)^{2 q+1}\left(\frac{\sin \sqrt{\lambda} t}{\sqrt{\lambda} t}\right)^{2 n-2 q-1} \\
& \cdot \sum_{c=0}^{q} \frac{((\tan \sqrt{\lambda} t) / \sqrt{\lambda})^{2 c}}{c !(2 c) ! 2^{c} \Gamma(n-q+c)} \int_{P} C^{c}\left(R^{P}-R^{\mathbf{C} P^{n}(\lambda)}\right) d P .
\end{aligned}
$$


Here $(d / d t) V_{P}^{\mathbf{C} P^{n}(\lambda)}(t)$ is the surface volume of the tube of radius $t$ about $P$. A proof of (4.7) using Jacobi fields is given in [GV]; proofs of (4.7) making use of the submersion $S^{2 n+1}(\lambda) \rightarrow \mathbf{C} P^{n}(\lambda)$ are given in [F] and [WO]. Formula (4.7) amounts to a Weyl tube formula for complex submanifolds of $\mathbf{C} P^{n}(\lambda)$. However, more is required for Theorem 1.1. The right-hand side of (4.7) must be expressed in terms of Chern forms and then the whole expression must be integrated from 0 to $r$.

Lemma 4.2. For $0 \leqslant t \leqslant$ the distance between $P$ and its nearest focal point,

$$
\begin{aligned}
& t^{2 n-2 q-1} \int_{S^{2 n-2 q-1}(1)} \theta_{u}(t) d U \\
& \quad=\frac{2 \pi \sin \sqrt{\lambda} t \cos \sqrt{\lambda} t}{(n-1) ! \sqrt{\lambda}}\left\langle\gamma\left(R^{P}-R^{\mathbf{C} P^{n}(\lambda)}\right) \wedge\left(\left(\frac{\pi}{\lambda}-F\right) \sin ^{2} \sqrt{\lambda} t+F\right)^{n-1}, \frac{1}{q !} F^{q}\right\rangle .
\end{aligned}
$$

Proof. It follows from (2.4) and (4.6) that

$$
\begin{aligned}
& \int_{S^{2 n-2 q-1}(1)} \theta_{u}(t) d u \\
& =\left(\frac{\sin \sqrt{\lambda} t}{\sqrt{\lambda} t}\right)^{2 n-2 q-1}(\cos \sqrt{\lambda} t)^{2 q+1} \int_{S^{2 n-2 q-1}(1)} \operatorname{det}\left(I-\frac{\tan \sqrt{\lambda} t}{\sqrt{\lambda}} T_{u}\right) d u \\
& =2 \pi^{n-q}\left(\frac{\sin \sqrt{\lambda} t}{\sqrt{\lambda} t}\right)^{2 n-2 q-1}(\cos \sqrt{\lambda} t)^{2 q+1} \sum_{c=0}^{q} \frac{C^{c}\left(R^{P}-R^{C P^{n}(\lambda)}\right)^{c}}{c !(2 c) ! 2^{c} \Gamma(n-q+c)}\left(\frac{\tan \sqrt{\lambda} t}{\sqrt{\lambda}}\right)^{2 c} .
\end{aligned}
$$

Hence from (3.1) and (4.9) follows

$$
\begin{aligned}
t^{2 n-} & =\int_{S^{2 n-2 q-1}(1)} \theta_{u}(t) d u \\
= & \frac{2 \pi(\sin \sqrt{\lambda} t)(\cos \sqrt{\lambda} t)^{2 n-1}}{\sqrt{\lambda}} \\
& \cdot \sum_{c=0}^{q} \frac{\left\langle\gamma_{c}\left(R^{P}-R^{\mathbf{C} P^{n}(\lambda)}\right) \wedge F^{q-c}, F^{q}\right\rangle}{(q-c) !(n-q+c-1) ! q !}\left(\frac{\pi \tan ^{2} \sqrt{\lambda} t}{\lambda}\right)^{n-q+c-1} \\
= & \frac{2 \pi(\sin \sqrt{\lambda} t)(\cos \sqrt{\lambda} t)^{2 n-1}}{(n-1) ! \sqrt{\lambda}} \\
& \cdot\left\langle\sum_{c=0}^{q} \gamma_{c}\left(R^{P}-R^{\mathbf{C} P^{n}(\lambda)}\right) \wedge \sum_{b=0}^{n-1}(n-1)\left(\frac{\pi \tan ^{2} \sqrt{\lambda t}}{\lambda}\right)^{n-b-1} F^{b}, \frac{1}{q !} F^{q}\right\rangle \\
= & \frac{2 \pi(\sin \sqrt{\lambda} t)(\cos \sqrt{\lambda} t)^{2 n-1}}{(n-1) ! \sqrt{\lambda}}\left\langle\gamma\left(R^{P}-R^{\mathbf{C} P^{n}(\lambda)}\right) \wedge\left(\frac{\pi}{\lambda} \tan ^{2} \sqrt{\lambda} t+F\right)^{n-1}, \frac{1}{q !} F^{q}\right\rangle .
\end{aligned}
$$

Hence (4.8) follows. 
COROllaRy 4.3. For $0 \leqslant r \leqslant$ distance from $P$ to its nearest focal point

$$
V_{P}^{\mathbf{C} P^{n}(\lambda)}(r)=\frac{1}{n !} \int_{P} \gamma\left(R^{P}-R^{\mathbf{C} P^{n}(\lambda)}\right) \wedge \frac{\left((\pi / \lambda) \sin ^{2} \sqrt{\lambda} r+F\right)^{n}}{1-(\lambda / \pi) F}
$$

Proof. When (4.8) is integrated from 0 to $r$ the result is

$$
\begin{aligned}
& \int_{0}^{r} \int_{S^{2 n-2 q-1}(1)} t^{2 n-2 q-1} \theta_{u}(t) d u d t \\
& \quad=\left\langle\gamma\left(R^{P}-R^{\mathbf{C} P^{u} n(\lambda)}\right) \wedge \frac{\left((\pi / \lambda) \sin ^{2} \sqrt{\lambda} r+\cos ^{2} \sqrt{\lambda} r F\right)^{n}}{1-(\lambda / \pi) F}, \frac{1}{q !} F^{q}\right\rangle .
\end{aligned}
$$

Hence (4.10) follows from (4.11) and (2.3)

Proof of TheOrem 1.1. Formula (1.1) is now an obvious consequence of Proposition 3.4 and Corollary 4.3.

5. Volumes of tubes about complete intersections. In this section let $P$ be a compact complex submanifold of $\mathbf{C} P^{n}(\lambda)$, and write $\gamma(t)=\hat{\gamma}\left(R^{P}\right)(t), \hat{\gamma}(t)=$ $\gamma\left(R^{P}\right)(t)$, etc.

Proof of Corollary 1.2. The total Chern class of a complete intersection $P^{a_{1} \cdots a_{n-a}}(\lambda) \subset \mathbf{C} P^{n}(\lambda)$ is given by

$$
[\gamma]=\left[\left(1+\frac{\lambda}{\pi} F\right)^{n+1} / \prod_{c=1}^{n-q}\left(1+\frac{a_{c} \lambda}{\pi} F\right)\right]
$$

(see for example [H, p. 159, K]). Hence

$$
[\gamma(t)]=\left[\left(1+\frac{t \lambda}{\pi} F\right)^{n+1} / \prod_{c=1}^{n-q}\left(1+\frac{t a_{c} \lambda}{\pi} F\right)\right]
$$

From (5.1) and Proposition 3.2 follows

$$
\begin{aligned}
{[\hat{\gamma}(t)] } & =\left[\left(1-\frac{\lambda}{\pi} F\right)^{q}\left(1+\frac{\lambda}{\pi} F\left(\frac{t}{1-(\lambda / \pi) F}\right)\right)^{n+1} / \prod_{c=1}^{n-q}\left(1+\frac{a_{c} \lambda}{\pi} F\left(\frac{t}{1-(\lambda / \pi) F}\right)\right)\right] \\
& \left.=\left[\left(1-\frac{\lambda}{\pi}(t-1) F\right)^{n+1} /\left(1-\frac{\lambda}{\pi} F\right) \prod_{c=1}^{n-q}\left(1-\frac{\left(t a_{c}-1\right) \lambda}{\pi} F\right)\right)\right] .
\end{aligned}
$$

In particular,

$$
[\hat{\gamma}(1)]=\left[\left(1-\frac{\lambda}{\pi} F\right) \prod_{c=1}^{n-q}\left(1+\frac{\left(a_{c}-1\right) \lambda}{\pi} F\right)\right]^{-1} .
$$

Now (1.3) follows from (5.2) and (1.1).

Proof of Corollary 1.3. When the right-hand side of (5.2) is expanded in a power series, the result is

$$
\begin{aligned}
& {\left[\left(1-\frac{\lambda}{\pi} F\right) \prod_{c=1}^{n-q}\left(1-\frac{\lambda\left(a_{c}-1\right)}{\pi} F\right)\right]^{-1}} \\
& =\left[\sum_{c=0}^{\infty}\left\{\prod_{i=1}^{n-q} \frac{1-\left(1-a_{i}\right)^{c+1}}{a_{i}}\right\}\left(\frac{\lambda}{\pi} F\right)^{c}\right] .
\end{aligned}
$$


From (5.3) follows the power series expansion for the integrand in the right-hand side of (1.3):

$$
\begin{aligned}
& {\left[\frac{\left((\pi / \lambda) \sin ^{2} \sqrt{\lambda} r+\cos ^{2} \sqrt{\lambda} F\right)^{n}}{n !(1-(\lambda / \pi) F) \Pi_{c=1}^{n-a}\left(1+\left(\lambda\left(a_{c}-1\right) / \pi\right) F\right)}\right]} \\
& \quad=\left[\left(\frac{\pi}{\lambda}\right)^{n} \sum_{b=0}^{\infty} \sum_{a=0}^{\min (b, n)} \frac{\left(\sin ^{2} \sqrt{\lambda} r\right)^{n-a}\left(\cos ^{2} \sqrt{\lambda} r\right)^{a}}{a !(n-a) !} f(b-a)\left(\frac{\lambda}{\pi} F\right)^{b}\right]
\end{aligned}
$$

where

$$
f(c)=\prod_{i=1}^{n-q}\left\{\frac{1-\left(1-a_{i}\right)^{c+1}}{a_{i}}\right\} .
$$

When (5.4) is integrated over $P$ all forms not of degree $2 q$ must be discarded. Hence from (5.4) and (2.3) follows

$$
V_{P}^{\mathbf{C} P^{n}(\lambda)}(r)=\left(\frac{\pi}{\lambda}\right)^{n} \sum_{a=0}^{q} \frac{\left(\sin ^{2} \sqrt{\lambda} r\right)^{n-a}\left(\cos ^{2} \sqrt{\lambda} r\right)^{a}}{a !(n-a) !} f(q-a)\left(\frac{\lambda}{\pi} F\right)^{q}[P] .
$$

Now (1.5) is a consequence of (1.4) and (5.5).

6. Comparison theorems. In this section it will be shown that there is a comparison theorem which simultaneously generalizes Theorem 1.1 and also the Bishop-Günther comparison theorem. This theorem is a refinement for Kähler submanifolds of the comparison theorems of [GR3].

The following notion will be needed:

Definition. Let $0 \leqslant \theta \leqslant 2 \pi$. Then the $\theta$-sectional curvature $K^{M}(\theta)$ of an almost Hermitian manifold $M$ is the restriction of the sectional curvature of $M$ to the 2-dimensional subspaces $\pi$ of tangent spaces which make an angle $\theta$ with a holomorphic section. (Thus $\pi$ has a basis of the form $x, \cos \zeta x+\sin \theta y$ where $y$ is perpendicular to $x$ and $J x$.) Then the holomorphic and antiholomorphic sectional curvatures are the cases $\theta=0$ and $\theta=\pi / 2$.

THEOREM 6.1. Let $P$ be a Kähler submanifold of a complete Kähler manifold $M$. Assume that $P$ is relatively compact and topologically embedded, and that $r>0$ is not larger than the distance from $P$ to its nearest focal point.

(i) Suppose $K^{M}(0) \geqslant 4 \lambda$ and $K^{M}(\pi / 2) \geqslant \lambda$. Then

$$
\begin{aligned}
V_{P}^{M}(r) & \leqslant \frac{1}{n !} \int_{P} \hat{\gamma}\left(R^{P}-R^{M}\right) \wedge\left(\frac{\pi}{\lambda} \sin ^{2} \sqrt{\lambda} r+\cos ^{2} \sqrt{\lambda} r\right)^{n} \\
& \leqslant \frac{1}{(n-q-1) !}\left(\frac{\pi}{\lambda} \sin ^{2} \sqrt{\lambda} r\right)^{n-q} \sum_{a=0}^{q}\left(\begin{array}{c}
q \\
a
\end{array}\right) \frac{\left(-\sin ^{2} \sqrt{\lambda} r\right)^{q}}{n-q+a} \operatorname{vol}(P) \\
& \leqslant \frac{1}{(n-q) !}\left(\frac{\pi}{\lambda} \sin ^{2} \sqrt{\lambda} r\right)^{n-q} \operatorname{vol}(P) .
\end{aligned}
$$

(ii) Suppose $K^{M}(0) \leqslant 4 \lambda$ and $K^{M}(\pi / 2) \leqslant \lambda$. Then

$$
V_{P}^{M}(r) \geqslant \frac{1}{n !} \int_{P} \hat{\gamma}\left(R^{P}-R^{M}\right) \wedge\left(\frac{\pi}{\lambda} \sin ^{2} \sqrt{\lambda} r+\cos ^{2} \sqrt{\lambda} r F\right)^{n} .
$$


Proof. The proof of Theorem 1.1 must be generalized. The four fundamental equations described in $\$ 2$ are completely general and so they may be used as they stand.

First part (i) will be established. Instead of (4.2) one has

$$
\left\{\begin{array}{l}
\kappa_{\alpha}^{\prime} \geqslant \kappa_{a}^{2}+\lambda, \quad \alpha \neq q+1,(q+1)^{*} \\
\kappa_{(q+1)^{*}}^{\prime} \geqslant \kappa_{(q+1)^{*}}^{2}+4 \lambda
\end{array}\right.
$$

and from (6.3) follows

$$
\begin{cases}\kappa_{a}(t) \geqslant-\frac{d}{d t} \ln \left(\cos \sqrt{\lambda} t-\frac{\kappa_{a}(0)}{\sqrt{\lambda}} \sin \sqrt{\lambda} t\right) & \text { for } a=1 \cdots q^{*}, \\ \kappa_{i}(t) \geqslant-\frac{d}{d t} \ln \sin \sqrt{\lambda} t & \text { for } i=q+2 \cdots n^{*}, \\ \kappa_{(q+1)^{*}}(t) \geqslant-\frac{d}{d t} \ln (\sin 2 \sqrt{\lambda} t) . & \end{cases}
$$

Then (6.4) implies

$$
\begin{aligned}
\operatorname{tr} S(t) \leqslant \frac{-d}{d t}\left\{\sum_{a=1}^{q^{*}} \ln \left(\cos \sqrt{\lambda} t-\frac{\kappa_{a}(0)}{\sqrt{\lambda}} \sin \sqrt{\lambda} t\right)\right. \\
\quad+2(n-q-1) \ln \sin \sqrt{\lambda} t+\ln \sin 2 \sqrt{\lambda} t\}
\end{aligned}
$$

From (6.5) follows the generalized version of (4.6):

$$
\theta_{u}(t) \leqslant\left(\frac{\sin \sqrt{\lambda} t}{\sqrt{\lambda} t}\right)^{2 n-2 q-1} \cos \sqrt{\lambda} t \prod_{a=1}^{q}\left(\cos ^{2} \sqrt{\lambda} t-\frac{\kappa_{a}(0)^{2}}{\lambda} \sin 2 \sqrt{\lambda} t\right) .
$$

It is clear that the rest of the proof goes as before with " $=$ " replaced by " $\leqslant$ " at the appropriate points. Thus the inequality corresponding to (4.8) is

$$
\begin{aligned}
& t^{2 n-2 q-1} \int_{S^{2 n-2 q-1}(1)} \theta_{u}(t) d u \\
& \quad \leqslant \frac{2 \pi \sin \sqrt{\lambda} t \cos \sqrt{\lambda} t}{(n-1) ! \sqrt{\lambda}}\left\langle\gamma\left(R^{P}-R^{C P^{n}(\lambda)}\right) \wedge\left(\left(\frac{\pi}{\lambda}-F\right) \sin ^{2} \sqrt{\lambda} t+F\right)^{n-1}, \frac{1}{q !} F^{q}\right\rangle .
\end{aligned}
$$

When (6.7) is integrated from 0 to $r$ and then over $P$ the result is the first inequality of (6.1).

To establish the second inequality in (6.1) let $\zeta_{u}(t)$ denote the quantity in the right-hand side of (6.6). It is clear that

$$
\zeta_{u}(t) \leqslant\left(\frac{\sin \sqrt{\lambda} t}{\sqrt{\lambda} t}\right)^{2 n-2 q-1}(\cos \sqrt{\lambda} t)^{2 q+1} \leqslant\left(\frac{\sin \sqrt{\lambda} t}{\sqrt{\lambda} t}\right)^{2 n-2 q-1} \cos \sqrt{\lambda} t .
$$


Thus

$$
\begin{aligned}
& \int_{0}^{r} t^{2 n-2 q-1} \int_{S^{2 n-2 q-1}(1)} \zeta_{u}(t) d u d t \\
& \quad \leqslant \frac{2 \pi^{n-q}}{\Gamma(n-q)} \int_{0}^{r}\left(\frac{\sin \sqrt{\lambda} t}{\sqrt{\lambda} t}\right)^{2 n-2 q-1}(\cos \sqrt{\lambda} t)^{2 q+1} d t \\
& \quad \leqslant \frac{\pi^{n-q}}{(n-q) !}\left(\frac{\pi}{\lambda} \sin ^{2} \sqrt{\lambda} r\right)^{n-q} .
\end{aligned}
$$

The integral in the middle term of (6.9) can be computed. Thus when (6.9) is integrated over $P$ and (6.6) is used, the second and third inequalities of (6.1) result.

The proof of (6.2) is similar to that of (6.1); this time all of the inequalities are reversed. The only difference is that there is no analog of (6.8).

\section{REFERENCES}

[BC] R. Bishop and R. Crittenden, Geometry of manifolds, Academic Press, New York, 1964.

[BGM] M. Berger, P. Gauduchon and E. Mazet, Le spectre d' une variètè riemannienne, Lecture Notes in Math., vol. 194, Springer-Verlag, 1971.

[F] F. J. Flaherty, The volume of a tube in complex projective space, Illinois J. Math. 16 (1972), 627-638.

[GR1] A. Gray, A generalization of F. Schur's theorem, J. Math. Soc. Japan 21 (1969), 454-457.

[GR2] __ Chern numbers and curvature, Amer. J. Math. 100 (1978), 463-476.

[GR3] Comparison theorems for the volumes of tubes as generalizations of the Weyl tube formula, Topology 21 (1982), 201-228.

[GR4] _ Volumes of tubes about Kähler submanifolds as expressed in terms of Chern classes, J. Math. Soc. Japan 36 (1983).

[GR5] _ A product formula for the volumes of tubes about Kähler submanifolds of complex Euclidean space, Riv. Mat. Univ. Parma (4) 104 (1984), 109-121 (volume in honor of E. Martinelli).

[GV] A. Gray and L. Vanhecke, The volumes of tubes in a Riemannian manifold, Rend. Sem. Mat. Univ. Politec. Torino 39 no. 3 (1981), 1-50.

[GS] P. A. Griffiths, Complex differential and integral geometry and curvature integrals associated to singularities on complex analytic varieties, Duke Math. J. 45 (1978), 427-472.

[GH] P. A. Griffiths and J. A. Harris, Principles of algebraic geometry, Wiley, 1978.

[H] F. Hirzebruch, Topological methods in algebraic geometry, (3rd ed.), Springer-Verlag, 1966.

[K] W. Katz, Die Chern-Formen von kählerschen Hyperflächen komplexer Raumformen, Inaugural Dissertation, Köln, 1979.

[MS] J.Milnor and J. Stasheff, Characteristic classes, Ann. of Math. Studies no. 76, Princeton Univ. Press, Princeton, N.J., 1974.

[MU] D. Mumford, Algebraic geometry. I, Complex projective varieties, Springer-Verlag, 1976.

[S] E. Study, Kürzeste Wege im komplexen Gebiete, Math. Ann. 60 (1905), 321-377.

[W] H. Weyl, On the volume of tubes, Amer. J. Math. 61 (1939), 461-472.

[WI] W. Wintinger, Eine Determinantenidentität und ihre Andwendungen auf analytische Gebilde in eukedischer und hermitescher Massbestimung, Monatsh. Math. und Physik 44 (1936), 343-365.

[WO] R. A. Wolf, The volume of tubes in complex projective space, Trans. Amer. Math. Soc. 157 (1971), $347-371$.

Department of Mathematics, University of Maryland, College Park, Maryland 20742 